\title{
LIST OF REVIEWERS VOLUME 22 (2013)
}

The Editors-in-Charge wish to thank the following reviewers:

\author{
Alatas Bilal \\ Alexandridis Alexandros \\ Anastassakis George \\ Apolloni Bruno \\ Bai Guohua \\ Bajo Javier \\ Banjade Rajendra \\ Bastide Rémi \\ Belesiotis Alexandros \\ Blekas Konstantinos \\ Caridakis Georgios \\ Chortaras Alexandros \\ Christodoulou Christodoulos \\ Ciaramella Angelo \\ Condotta Jean-François \\ Culotta Aron \\ Dafli Eleni \\ Daradoumis Thanasis \\ Datla Vivek \\ Delibasis Kostas \\ Dong Yulin \\ Franca Manoel \\ Foteinos Antonis \\ Garcez Artur d'Avila \\ García-Pedrajas Nicolás \\ Garofalakis John \\ Gkotsis George \\ Gkoudalas-Divanis Aris \\ Hatzilygeroudis Ioannis \\ Hermoso Ramon
}

\author{
Huang Yitzen \\ Ion Radu \\ Isern David \\ Jarrar Radi \\ Kabassi Katerina \\ Kolomvatsos Kostas \\ Kanellopoulos Yiannis \\ Karaboga Dervis \\ Karatsiolis Savvas \\ Kossida Sophia \\ Kotsiantis Sotiris \\ Koukoumialos Stylianos \\ Koutkias Vassilis \\ Koutras Vassilios \\ Kuhn Lisa \\ Leoni Massimiliano De \\ Lhotska Lenka \\ Lin Jung-Hsin \\ Lu Meiliu \\ Magoulas George \\ Makris Christos \\ Mani Nariman \\ Maragoudakis Manolis \\ Margaritis Konstantinos \\ Marinakis Yannis \\ Markov Zdravko \\ Mavroudi Seferina \\ Megalooikonomou Vasilis \\ Mileo Alessandra \\ Moradi Amirmohammad
}


Moreno Antonio

Neocleous Costas

Nguifo Engelbert Mephu

Nogueira Monica

Orlov Nikita

Pantelopoulos Alexandros

Papadopoulos Harris

Paris Lionel

Patkos Theodore

Patsakis Constantinos

Pattichis Constantinos

Pissis Solon

Poulas Konstantinos

Prentzas Jim

Psomopoulos Evangelos

Rajpal P.S.

Rodriguez Yanet

Rus Vasile

Russell Steele

Santamaria-Pang Alberto

Sgarbas Kyriakos

Sioutas Spyros
Song Minseok

Spanakis Gerasimos

Stafylopatis Andreas

Stamou Georgios

Jun Sun

Tambouratzis Tatiana

Theodoridis Evangelos

Theoharatos Christos

Thomaidis Nikos

Thomaz Carlos Eduardo

Tzimas Giannis

Valls Aida

Varga Laszlo

Vázquez-Salceda Javier

Wang Junxian

Wessel Michael

$\mathrm{Wu}$ Yi-Hung

Xiao Liangliang

York-Smith Neil

Zacharaki Evaggelia

Zhang Nan

Zhou Guang 